\title{
Clozapine $v$. chlorpromazine in treatment-naive, first-episode schizophrenia: 9-year outcomes of a randomised clinical trial ${ }^{* \dagger}$
}

Ragy R. Girgis, Michael R. Phillips, Xiaodong Li, Kejin Li, Huiping Jiang, Chengjing Wu, Naihua Duan, Yajuan Niu and Jeffrey A. Lieberman

\section{Background}

The differential effects of so-called 'first- and secondgeneration' antipsychotic medications, when given in the first episode, on the long-term outcome of schizophrenia remain to be elucidated.

\section{Aims \\ We compared the 9-year outcomes of individuals initially randomised to clozapine or chlorpromazine.}

\section{Method}

One-hundred and sixty individuals with treatment-naive, firstepisode schizophrenia or schizophreniform disorder in a mental health centre in Beijing, China were randomised to clozapine or chlorpromazine treatment for up to 2 years, followed by up to an additional 7 years of naturalistic treatment. The primary outcome was remission status for individuals in each group.

\section{Results}

Individuals in both groups spent essentially equal amounts of time in each clinical state over the follow-up time period (remission, 78\%; intermediate, 8\%; relapse, 14\%). There were no significant differences on other measures of illness severity. The clozapine group was more likely than the chlorpromazine group to remain on the medication to which they were originally assigned $(26 \%$ v. $10 \%, P=0.01)$. There were no significant differences between the two groups on other secondary efficacy outcomes.

\section{Conclusions}

These findings support the comparability in effectiveness between antipsychotic medications but with slightly greater tolerability of clozapine in the treatment of first-episode psychosis.

\section{Declaration of interest}

R.R.G. has received research support from Janssen and Lilly through APIRE and a travel stipend from Lilly, Forest, and Elsevier Science through the Society of Biological Psychiatry. N.D. has received research support from Pfizer. J.A.L. has received research grant support from Acadia, Allon, AstraZeneca, Bristol-Myers Squibb, Forest Labs, GlaxoSmithKline, Janssen, Merck, Organon, Pfizer, and Wyeth. He has acted as a consultant and an advisory board member for Astra-Zeneca, Bristol-Myers Squibb, GlaxoSmithKline, Eli Lilly, and Pfizer; has been a consultant for Cephalon, Johnson \& Johnson and Novartis; an advisory board member for Bioline, Forest Labs, Lundbeck, Organon and Wyeth; and a DSMB member for Solvay. J.A.L. has received no direct financial compensation or salary support for participation in research, consulting, advisory board or DSMB activities. J.A.L. holds a patent from Repligen.
Recent research has shown that antipsychotic medications may be able to affect the natural course of schizophrenia, ${ }^{1,2}$ particularly when given early during the course of the illness. ${ }^{3-6}$ This suggests that antipsychotic medications may prevent disease progression in schizophrenia by a mechanism that is yet to be identified. ${ }^{7,8}$

Acute-phase efficacy results suggest that few differences in therapeutic efficacy exist between individual or classes of antipsychotic medications. ${ }^{9-13}$ Fewer studies have examined the comparative effectiveness of antipsychotic drugs in first-episode psychosis and whether the initial exposure to one medication or another will differentially affect long-term outcome. ${ }^{14-17}$

The ongoing debate about the relative merits of different classes (for example first- $v$. second-generation antipsychotics) or individual antipsychotic drugs has been waged in chronic, ${ }^{18,19}$ first-episode, ${ }^{9-17}$ treatment-refractory ${ }^{20}$ and adolescent ${ }^{21}$ populations. Despite the fact that there are marked

*Aspects of this study have been presented at the 2009 Annual Meeting of the American Psychiatric Association (San Francisco, USA, 17 May), the 2009 Annual Meeting of the New York County District Branch of the American Psychiatric Association (New York, USA, 30 April) and the 11th Annual Symposium on Statistics in Psychiatry (Philadelphia, USA, 11 May 2009).

†See editorials, pp. 266-268 and 269-271, this issue. differences in opinions and that the validity of the so-called classes of antipsychotic drugs has been questioned, ${ }^{22}$ the predominant impression is that there are minimal differences across patient groups, except that clozapine (and to a lesser extent olanzapine $e^{23,24}$ ) is superior in treatment-refractory illness.

Given the superiority of clozapine to other antipsychotic drugs in refractory illness, we hypothesised that its superior effect would also be manifest in first-episode schizophrenia. We tested this hypothesis in a sample of first-episode, antipsychotic-naive individuals by randomising them to clozapine or chlorpromazine with long-term follow-up. The 52-week outcome results have been reported previously: we found that treatment with clozapine led to greater efficacy after 12 weeks of in-patient treatment, faster time to remission and greater time in remission, however, by 52 weeks these efficacy differences were no longer observed. ${ }^{25}$ No outcome results from that trial beyond 1 year have been reported. In this paper, we report the 9-year follow-up results. We hypothesised that individuals originally randomised to clozapine as their first treatment exposure would have better long-term outcomes than those who were originally randomised to chlorpromazine, with the understanding that the majority of individuals would probably have taken several medications other than clozapine or chlorpromazine by the end of the study. 


\section{Method}

\section{Participants}

A detailed description of the design for this study has been published previously. ${ }^{25}$ This study was designed by the senior authors (J.A.L. and M.R.P.) and conducted at the Beijing Suicide Research and Prevention Center at the Beijing Hui Long Guan Hospital. It was approved by the Research Review Board of the Beijing Bureau of Health and conformed to the international standards for research ethics. All hospital admissions between October 1995 and December 1998 were screened for inclusion in this study. Inclusion criteria were: age between 16 and 40 years, diagnosis of schizophrenia or schizophreniform disorder, no previous antipsychotic treatment or a maximum of 14 days of prior use, maximum symptom duration of 60 months, current psychotic symptoms of moderate severity or greater for at least one of the five psychotic items assessed by the Brief Psychiatric Rating Scale (BPRS). ${ }^{26}$ Written informed consent was provided by individuals and their families for those individuals who met the preceding criteria.

Diagnoses were established with a Chinese version of the Structured Clinical Interview for DSM-IV Axis I disorders, ${ }^{27}$ along with blood tests and physical examinations. Participants were initially randomised to either clozapine with a placebo benztropine or chlorpromazine with benztropine $(2 \mathrm{mg}$ twice daily) while in the hospital. For randomisation, the study nurse stratified eligible in-patients into six groups by gender and duration of positive symptoms (3-9 months, 9-24 months and 24-60 months). Participants in each of these six strata were randomised in blocks of four to either the clozapine or chlorpromazine treatment group. Participants remained in the hospital for 12 weeks, after which they were followed monthly as out-patients. They were maintained on their assigned medication under double-blind conditions for 2 years or up to the point that there was a clinical indication to change their medication regimen, whichever came first. After this point all participants were treated in an open-label, naturalistic manner. Treatment adherence was monitored via pill counting during both the randomised and open-label phases of the study.

\section{Assessments}

Efficacy assessments were performed at study entry, weekly for weeks 1-6, biweekly for weeks 6-12, at hospital discharge and every 3 months thereafter. Assessments included the Chinese versions of the BPRS, ${ }^{26}$ the Scale for the Assessment of Negative Symptoms (SANS), ${ }^{28}$ the Clinical Global Impression Scale $(\mathrm{CGI})^{29}$ and the Global Assessment of Functioning Scale (GAF). ${ }^{30}$ To assess side-effects, we used the Simpson Angus Extrapyramidal Symptoms Scale (SAESS) ${ }^{31}$ (omitting sialorrhea given the use of clozapine in this trial) on the same schedule for the first 2 years and then every 6 months thereafter; and classification terms from the Coding Symbol and Thesaurus for Adverse Event Terminology (COSTART) at each assessment visit for the first year, every 3 months for the second and third years, and every 6 months thereafter. We assessed for tardive dyskinesia using the Tardive Dyskinesia Rating Scale. ${ }^{32}$ At periodic intervals two clinicians independently assessed participants, which allowed for verification of ratings. Electrocardiograms (ECGs) were performed at study entry, weeks 12 and 52 and yearly thereafter. White blood cell counts were done weekly during the in-patient phase of the study (i.e. approximately 12 weeks), monthly for the remainder of year 1 and year 2, and every 3 months thereafter. Fasting blood glucose values were assessed at study entry, week 12 , every 3 months for the remainder of the first year and every six months thereafter. Weight was not regularly assessed during this study, so our analysis (see statistical methods below) only included weights for the 29 participants for whom we were able to calculate weight change. Additionally, the occurrence of seizures was not formally assessed during this study. However, none was reported in either group.

\section{Statistical methods}

Our primary analysis compared treatment efficacy for clozapine $v$. chlorpromazine for schizophrenia, following the intent-to-treat principle to compare patient outcomes according to the initial treatment assignment. Our primary analytical strategy employed generalised linear mixed models (GLMMs) for categorical repeated measures such as remission status, and mixed-effect models for continuous repeated measures such as the BPRS, SANS, CGI and GAF. The inference from these models is valid provided that the missing data are 'missing at random. ${ }^{33}$ The programs PROC GLIMMIX and MIXED in SAS (version 9.1.3) for Windows were used to estimate and test these models.

Following our previous publication from this study, ${ }^{25}$ the primary outcome measure was participant's remission status over the course of the 9-year observation period, classified into three ad hoc clinical states: remission, intermediate state and relapse. Remission was defined as a reduction in the total BPRS score of $50 \%$ or more from baseline, with scores of mild (3) or less on all five BPRS psychotic items (unusual thought content, suspiciousness, hallucinations, conceptual disorganisation, mannerisms and posturing) and a CGI-Severity item of mild (3) or less. Relapse was defined as having at least one BPRS psychotic item scored moderately severe (5) or higher, or at least two BPRS psychotic items scored moderate (4) or higher. The intermediate state was defined as everything else.

A three-category GLMM with logistic links was specified for the remission status as a function of initial treatment assignment, time, and other baseline covariates: baseline BPRS, gender, duration of untreated psychosis (DUP) and age at onset of psychosis. The effect of initial treatment assignment on the secondary outcome measures, including BPRS, SANS, CGI and GAF, which were all treated as continuous measures, were estimated and tested using an approach similar to that described above.

The effect of initial treatment assignment on participants' time to drop out (retention) and the time to stopping study medication was examined using Kaplan-Meier estimates for the respective survival curves, and tested using log-rank tests. This analysis was expanded further using a Cox proportional hazards model to adjust for the covariates listed above. We used Fisher's exact test to compare the proportions of individuals in each group who remained in the study after 9 years, the proportions of individuals in each group who remained on the originally assigned study medication, and the proportion of individuals who switched from chlorpromazine to clozapine or vice versa. Individuals who stopped taking their originally assigned medication for less than 3 months and then resumed the same medication were not considered to have discontinued their study medication unless they were treated with another antipsychotic medication.

We used $t$-tests to compare the average percentage of time on any antipsychotic medication after the first year, the average dose of antipsychotic medications in terms of chlorpromazine equivalents between year 2 and year 9, and the average dose of antipsychotic medications after the first year during days on which antipsychotic medications were taken (clozapine doses were converted to chlorpromazine equivalents by multiplying the clozapine dose by 1.33). The effect of cumulative antipsychotic 
dosage on the improvement of the BPRS from year 2 through year 9 or drop-out was compared for the two treatments using a linear regression model adjusted for several baseline covariates: baseline BPRS score, participant gender, DUP and age at onset of psychosis. We also examined the average percentages of time that individuals in each group took various antipsychotic medications after discontinuing the originally assigned study medication.

We used Fisher's exact test to compare the proportions of participants who developed tardive dyskinesia and agranulocytosis (absolute neutrophil count $<500$ ) for the two treatment groups, and the proportion of individuals who developed tardive dyskinesia, along with mean weight gain, for participants who remained on the originally assigned study medications for 9 years. Tardive dyskinesia was liberally defined as a score of $\geqslant 2$ on any body part subscale from a version of the Tardive Dyskinesia Rating Scale. ${ }^{32}$

Analysis of covariance (ANCOVA) models were used to test the differences in laboratory parameters at the end of the study (percentage of neutrophils and lymphocytes, white blood cells, glucose, heart rate and ECG QT interval) between the two treatments after controlling for baseline BPRS, participant gender, DUP and age at onset of psychosis. Some of the laboratory measures were not collected at the end of the study, so we filled in the missing values ( $n=5$ for weight gain and $n=1$ for glucose) with regression-based extrapolations. Finally, for demographic and clinical variables, Fisher's exact test was used for categorical variables and $t$-test was used for continuous variables. All tests used a two-sided alpha-level of 0.05 .

\section{Results}

\section{Participants}

The demographic and clinical data for the participants in this study have been previously published. ${ }^{25}$ Briefly, there were no statistically significant differences between the participants in the clozapine and chlorpromazine groups on any demographic variable (i.e. diagnosis, age, gender, baseline symptom severity and duration, and age at onset of psychotic symptoms; see online Table DS1 for the between-group analyses for those variables used later in this analysis for which results were adjusted). The average age of participants at study entry was 28.7 years $($ s.d. $=6.9)$ with a range of 15 to 42 . The majority of individuals were diagnosed with paranoid schizophrenia (53\%), and the rest with either undifferentiated schizophrenia $(23 \%)$ or schizophreniform disorder (24\%). Participants experienced psychotic symptoms for an average of 18.4 months (s.d. $=17.8$ months, median 10.7) at the time of study entry. The mean age at onset of psychotic symptoms was 27.2 years (s.d. $=6.5$ years, median 26.9 ). The mean baseline symptom severity scores were 43.8 (s.d.=5.1) for total BPRS, 5.6 (s.d. =0.6) for CGI-severity and 35.8 (s.d.=7.8) for GAF.

\section{Retention and treatment status}

The first-year outcome data have been reported previously. ${ }^{25}$ Figure 1 presents the flow of participants in this study. Of the 160 participants who initially received treatment with clozapine $(n=80)$ or chlorpromazine $(n=80), 124(77.5 \%)$ continued to be followed in the study for 9 years, 63 in the clozapine group (79\%) and 61 in the chlorpromazine group $(76 \%)(P=0.85)$. There was no statistically significant difference in time to drop out between the two groups $(P=0.71)$ (Fig. 2). This outcome remained statistically insignificant after using a Cox proportional hazard regression analysis to adjust for baseline total BPRS score, age at onset of psychotic symptoms, gender and DUP. Of the 36 individuals who dropped out of the study during the 9-year period, 19 (clozapine 9; chlorpromazine 10) dropped out during the first year. (Notably, this result is different from that reported in the initial report ${ }^{25}$ because in the current analysis drop-out was defined as leaving the study, whereas in the original analysis drop-out included individuals who discontinued the medication to which they were originally randomised but continued to be followed-up by study staff.) Reasons for drop-out from the study included: for the clozapine group - withdrew consent 6 , adverse effects 1 , lack of efficacy 0 , death 2 , lost to follow-up 7, elopement from the hospital 1, imprisonment 0 ; for the chlorpromazine group - withdrew consent 6 , adverse effects 1 , lack of efficacy 1 , death 2, lost to follow-up 8, elopement from the hospital 0 , imprisonment 1 . The causes of the four deaths were viral encephalitis (or meningitis), accident, unknown illness and suicide. Overall, the mortality rates were $2.5 \%(2 / 80)$ in both treatment groups.

Twenty-nine participants (18\%) remained on the originally assigned medication after 9 years, 21 in the clozapine group $(26 \%)$ and 8 in the chlorpromazine group $(10 \%)(P=0.01)$. The median amount of time until first discontinuation of the originally assigned study medication was 39 months in the clozapine group and 23 months in the chlorpromazine group, a statistically significant advantage for clozapine (log-rank 7.49, d.f. $=1, P=0.01$ ) (Fig. 3). The result was similar when examined using a Cox proportional hazard regression to adjust for baseline total BPRS score, age at onset of psychotic symptoms, gender and DUP (hazard ratio $0.644,95 \%$ CI $0.45-0.92, P=0.01$ ). Among individuals who remained in the study after 1 year $(n=70$ for clozapine, 69 for chlorpromazine), there was a non-significant trend for those in the clozapine group to spend more time (in terms of cumulative percentage of days) on any antipsychotic medication than those in the chlorpromazine group $(77 \% \mathrm{v}$. $66 \%, t(137)=-1.82, P=0.07)$, whereas the average doses of any antipsychotic medications, in terms of chlorpromazine equivalents, used by patients were similar (219v. 206, respectively, $t(137)=-0.49, P=0.62)$. The difference remained non-significant when the analysis was limited to days on which antipsychotic medications were taken (291 v. 319, respectively; $t(126)=1.08$, $P=0.28 ; n=65$ for clozapine and $n=63$ for chlorpromazine). Additionally, there were no significant demographic or baseline clinical differences between the group of participants who remained on their originally assigned medication for the duration of the study $(n=29)$ and the group of participants who remained in the study for 9 years and switched medications $(n=95)$ (online Table DS2).

\section{Efficacy}

As previously reported, ${ }^{25}$ the clozapine group spent significantly more time in remission during the first year of the study and had a faster time to remission in terms of psychopathological response during the first year of treatment. However, the average percentage of time that individuals spent in the three clinical states from years 2 through to 9 were essentially identical for the clozapine and chlorpromazine groups: 78\% (remission), $8 \%$ (intermediate) and $14 \%$ (relapse) (Fig. 4). There were no statistically significant differences across groups in the average percentages of time spent in each clinical state or on any efficacy measure (i.e. BPRS, SANS, CGI-Severity, GAF), either averaged over the entire study time period (i.e. years 2 through to 9) or at any individual follow-up time point (for example year 2, year 3) (Table 1). These results remained statistically non-significant after adjusting for baseline BPRS score, age at onset of psychotic symptoms, gender and DUP. In addition, there was no significant 


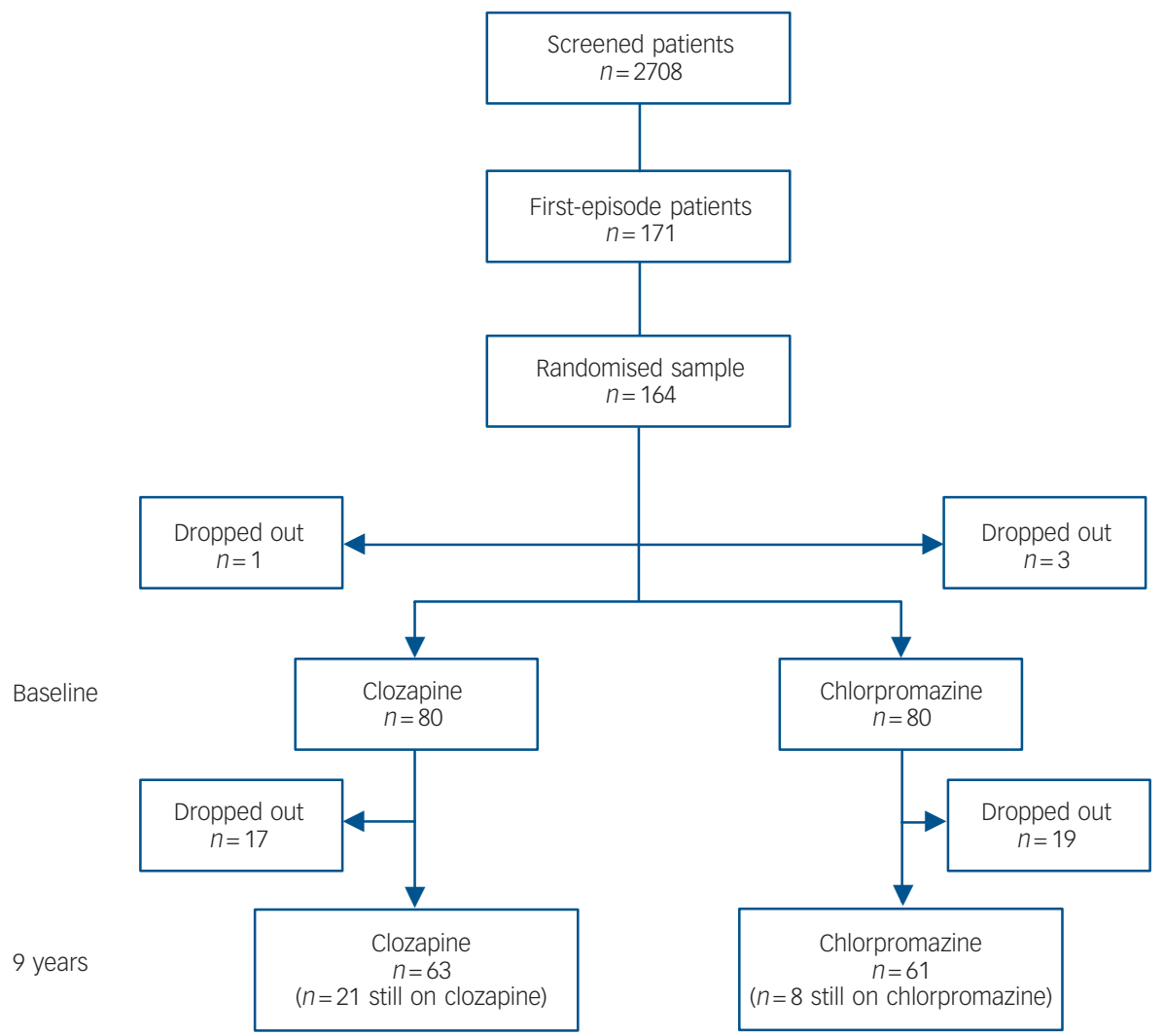

Fig. 1 Consort diagram.

effect of cumulative antipsychotic dose on improvement on the BPRS ( $n=139, t(1)=-0.07, P=0.95)$ nor was there an interaction effect between drug group (i.e. clozapine or chlorpromazine) and cumulative dosage $(t(1)=-0.02, P=0.98)$.

As a result of the similarities on outcome and efficacy measures between the clozapine and chlorpromazine groups, additional analyses were performed to determine the percentages of participants from the chlorpromazine group who took

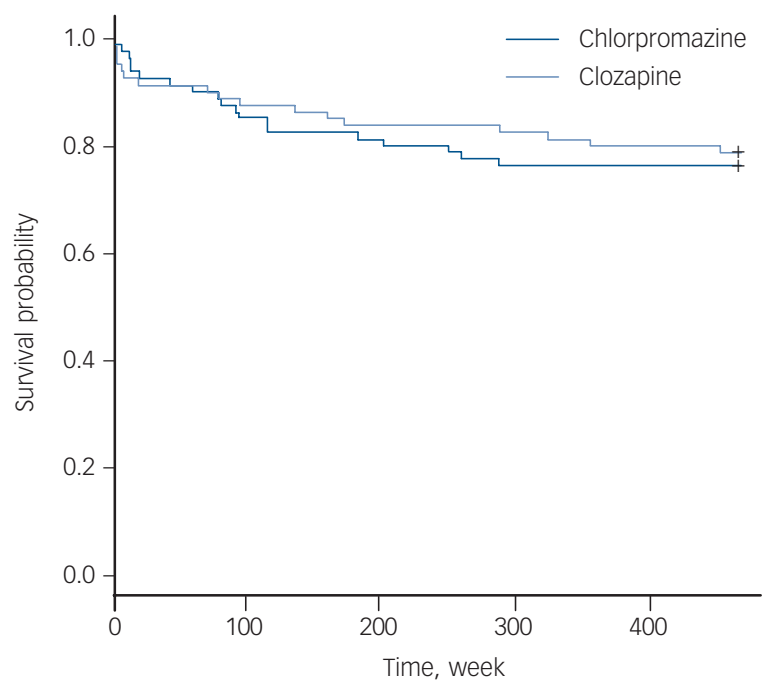

Fig. 2 Kaplan-Meier survival curves for time to drop out from the study for the clozapine and chlorpromazine groups. There is no statistically significant difference between the two groups $(P=0.71)$ clozapine at some point during the 9-year study, and vice versa. Among individuals originally randomised to chlorpromazine, $30 \%(24 / 80)$ took clozapine at some point during the study; only $3.8 \%(3 / 80)$ of the clozapine group took chlorpromazine at some point during the study $(P<0.01)$. In addition, we determined the average percentages of time that participants in each group took other antipsychotic medications or resumed the original medication, and report them here descriptively: after discontinuing

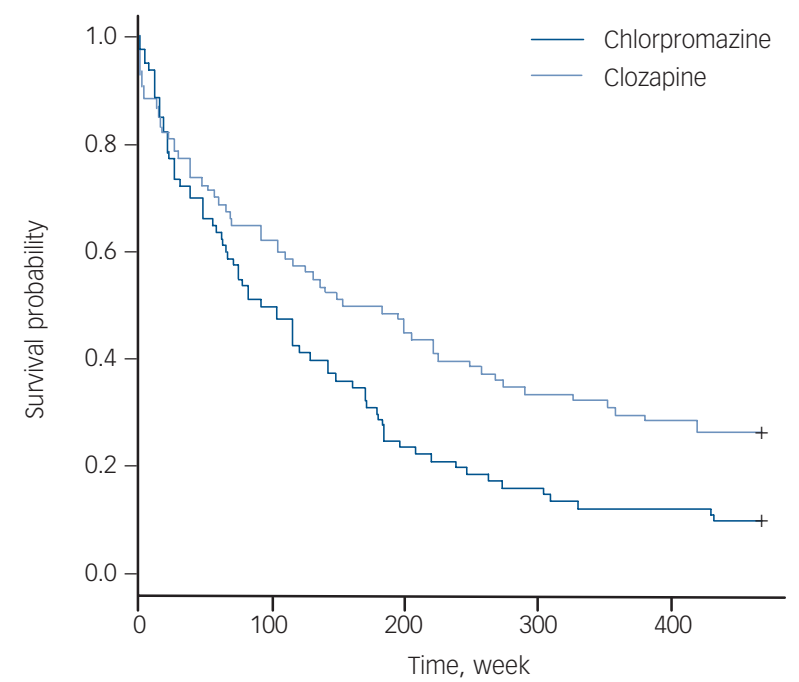

Fig. 3 Kaplan-Meier survival curves for time to first discontinuation of the original study medication for the clozapine and chlorpromazine groups. There is a statistically significant advantage for clozapine $(P=0.01)$. 
(a)

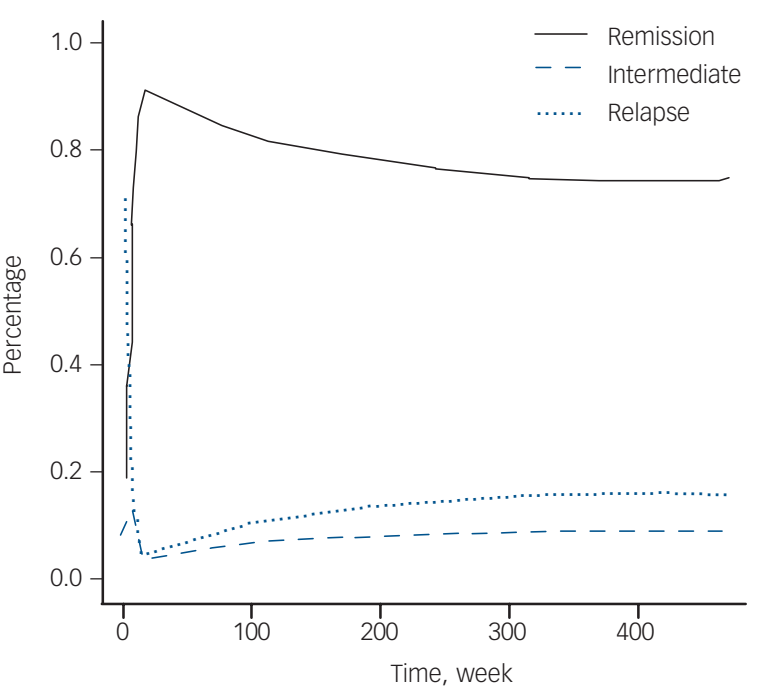

(b)

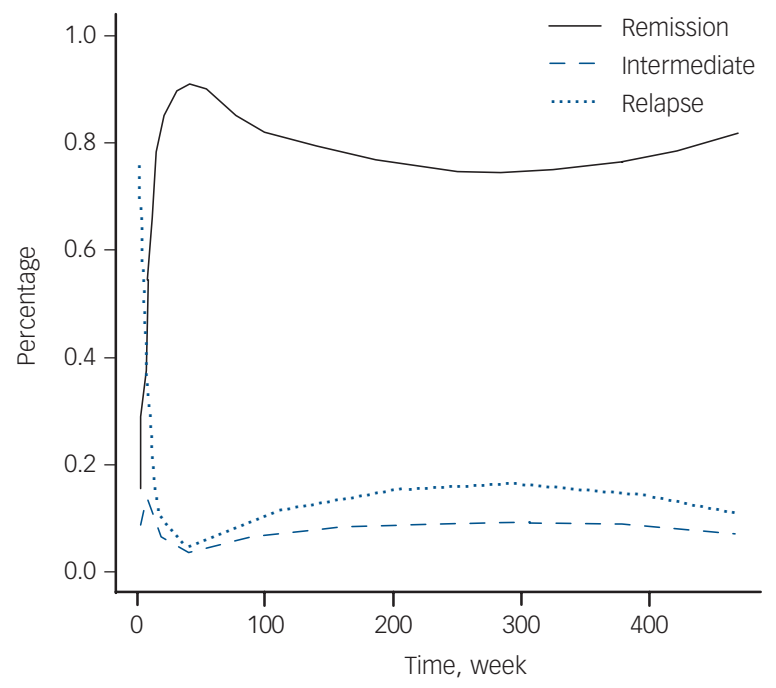

Fig. 4 Curves showing the per cent of individuals in the (a) clozapine and (b) chlorpromazine groups who were in the remission (solid line), intermediate (dashed line), or relapsed (dotted line) states throughout the 9-year study period. These curves are essentially identical.

chlorpromazine ( $n=72$, median days 2091), chlorpromazine $2 \%$, clozapine $13 \%$, another first-generation antipsychotic $4 \%$, another second-generation antipsychotic $19 \%$, unspecified antipsychotic $<1 \%$, multiple antipsychotics $7 \%$, no antipsychotic $55 \%$; after discontinuing clozapine $(n=59$, median days 1895$)$, chlorpromazine $<1 \%$, clozapine $7 \%$, another first-generation antipsychotic 6\%, another second-generation antipsychotic 13\%, unspecified antipsychotic $<1 \%$, multiple antipsychotics $1 \%$, no antipsychotic $73 \%$.

\section{Safety}

Of 160 participants enrolled in the study, four (2.5\%) developed agranulocytosis (two were randomised to clozapine and two were randomised to chlorpromazine). At the time that their agranulocytosis developed, one of these individuals was taking clozapine alone, one was taking chlorpromazine and trihexyphenidyl, and one was taking risperidone. The fourth person developed agranulocytosis while on aripiprazole and

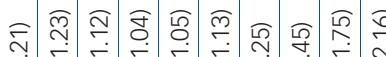

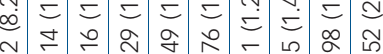

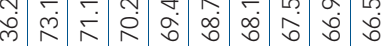

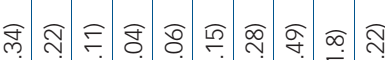

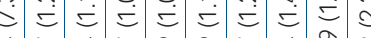

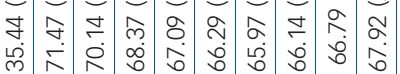

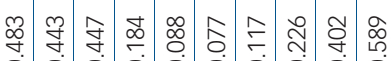
○.

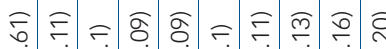

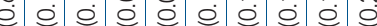
นٌ

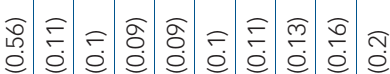
ᄃ.

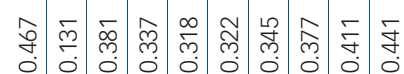

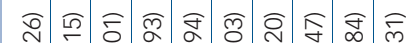

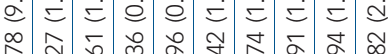

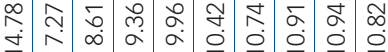

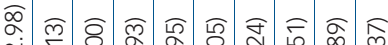

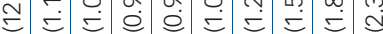
৪

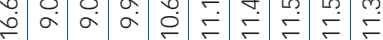

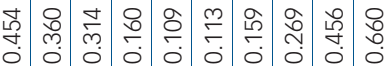

จ ○ 0000000 ป m্ণ

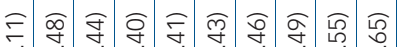
ए)

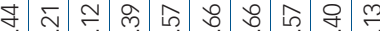

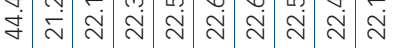


clonazepam, and then while on clozapine along with aripiprazole and propranolol. The white blood cell counts of all of these people returned to normal with appropriate modification of their medications.

Of the 160 participants, a total of $26(16 \%)$ developed tardive dyskinesia. Nine $(11.3 \%)$ of these were randomised to clozapine and $17(21.3 \%)$ were randomised to chlorpromazine $(P=0.02)$. Of the 21 individuals who remained on clozapine and the 8 who remained on chlorpromazine for the entire 9 years of this study, one person on clozapine (4.8\%) developed tardive dyskinesia, as did two participants on chlorpromazine (25\%) $(P=0.18)$. Each of these three participants was on antipsychotic monotherapy with either clozapine or chlorpromazine for the entire study period before development of their tardive dyskinesia, and one of the individuals in the chlorpromazine group had taken lorazepam, chlorpheniramine and benzhexol, each for 1-2 months, approximately 2 years before development of tardive dyskinesia.

Among the 29 participants who remained on either clozapine $(n=21)$ or chlorpromazine $(n=8)$ for the complete study, there were no significant differences in weight gain (clozapine $11.39 \mathrm{~kg}$, chlorpromazine $12.74 \mathrm{~kg}, P=0.79$ ), white blood cell count (clozapine 5933, chlorpromazine 5225, $P=0.28$ ), per cent of neutrophils (clozapine 64.3, chlorpromazine 62.4, $P=0.73$ ), per cent of lymphocytes (clozapine 31.3, chlorpromazine 32.5, $P=0.82$ ), ECG heart rate (clozapine 85, chlorpromazine 79 , $P=0.49$ ), ECG QT interval (clozapine 0.34, chlorpromazine $0.34, P=0.98$ ) or fasting glucose level (clozapine $6.8 \mathrm{mmol} / \mathrm{l}$, chlorpromazine $5.8 \mathrm{mmol} / \mathrm{l}, P=0.21$ ) at the 9 -year end-point.

\section{Discussion}

\section{Main findings}

This study extends the paper by Lieberman et $a l^{25}$ on the first year of randomised treatment to either clozapine or chlorpromazine of 160 individuals with first-episode psychosis. In contrast to our hypothesis, we found that the initial exposure of individuals with first-episode psychosis to either chlorpromazine or clozapine did not alter the long-term outcome of their illness. Clozapine and chlorpromazine do not appear to have substantially differential effects on the long-term course of schizophrenia, and presumably therapeutic efficacy, when used as the initial treatment strategies in the first episode. Outcomes in individuals treated initially with clozapine and chlorpromazine were essentially identical on the primary outcome variable, remission status, and demonstrated minimal differences on all measures of psychopathology, including negative symptoms, at every follow-up time period (Table 1). The only differences in outcome were seen in treatment continuation and retention, and these are believed to reflect the relative tolerability of the medications. These results are consistent with the results reported for the first year of this study in that the advantages seen at 12 weeks for clozapine were not sustained at 52 weeks $^{25}$ or thereafter. Finally, there was no effect of cumulative antipsychotic dosage on long-term outcome in this study.

The design of this study systematically addressed the question of whether clozapine has a putative disease-modifying effect (possibly neuroprotective) when administered in the first psychotic episode and confers a lasting value to individuals in the subsequent course of their illness. These results suggest that it is equally effective to wait until individuals demonstrate themselves to be non- or poorly responsive to antipsychotic medications before employing clozapine in first-episode patients, ${ }^{34}$ rather than using it prophylactically. This finding remained unchanged throughout the randomised, double-blind portion of the study (i.e. through 2 years) and for the subsequent 7-year period of naturalistic treatment, at every follow-up time period (Table 1). Although more individuals stayed on clozapine for the extent of the study, this was a small proportion of the first-episode participants initially treated with clozapine and did not translate into clinical advantages, despite the overall high retention and good antipsychotic exposure in this study. These results are consistent with the findings from CATIE, ${ }^{18}$ CUtLASS $^{19}$ and EUFEST $^{17}$ that found few substantial differences in effectiveness between second- and first-generation antipsychotics in non-refractory illness, and therefore add to the body of evidence tempering the assertions that second-generation antipsychotics are greatly superior to first-generation ones.

It is notable that nearly $80 \%$ of individuals remained in the study in open, naturalistic treatment with any of a variety of antipsychotic medications and on appropriate dosages (averages of $219 \mathrm{mg} / \mathrm{day}$, in terms of chlorpromazine equivalents, in the clozapine group and $206 \mathrm{mg} /$ day in the chlorpromazine group over the entire maintenance period) for 9 years. Furthermore, these individuals were in the remitted state for approximately $78 \%$ of that time - a remarkable outcome, even in the era of antipsychotic medications. ${ }^{35,36}$ This may be related to the medication adherence achieved during this study and is consistent with previous results that report that medication adherence is critical for the relief of symptoms in first-episode patients, ${ }^{37}$ including data from one trial showing an almost fivefold greater chance of relapse when medications are discontinued. ${ }^{36}$ This may also be related to the active involvement of the staff at the Beijing Suicide Research and Prevention Center who maintained close contact with participants and their families - so that medications could be restarted quickly if the individual's condition deteriorated. The value of such psychosocial interventions has been demonstrated and documented ${ }^{38,39}$ and cannot be overstated. These results also demonstrate what can be accomplished in settings where retention can be maintained, which is, unfortunately, not available to the majority of individuals with schizophrenia and therefore limits the generalisability of these findings.

\section{Side-effects}

Among the 29 individuals who remained on clozapine or chlorpromazine for the entire 9 years of the study, rates of tardive dyskinesia were greater in the chlorpromazine than in the clozapine group, as expected, ${ }^{40}$ although it should be noted that this difference was not statistically significant, likely the result of the small sample size in this subsample. Second, agranulocytosis occurred only once among individuals treated with clozapine monotherapy, consistent with previous reports of the prevalence of agranulocytosis (approximately 1\%). ${ }^{41}$ Finally, the degree of metabolic and other side-effects were similar between the two groups, which is consistent with the data on clozapine and chlorpromazine, both of which are known to induce metabolic effects. ${ }^{42,43}$

\section{Limitations}

There are several limitations to this study. Participants were in open, naturalistic treatment for the majority of the follow-up period after initially receiving randomised, double-blind treatment, and there was notable crossover between the two groups. This naturalistic treatment may also have confounded the effect of dosing on long-term outcome. 


\section{Implications}

In terms of the question, does the type of antipsychotic medication to which a patient is first exposed influence their long-term outcome, regardless of the type of treatment to which they are later exposed, the answer from this study appears to be no. To the extent that there are any differences, these appear to be related to side-effects and tolerability and not therapeutic efficacy. The findings from this 9-year investigation of treatment-naive, first-episode participants originally randomised to clozapine or chlorpromazine suggest that the long-term outcomes of individuals with first-episode psychosis are no different whether their initial exposure is to clozapine or chlorpromazine. Further, these findings support the growing body of literature that shows few differences in effectiveness between antipsychotic medications in non-treatment refractory schizophrenia.

\footnotetext{
Ragy R. Girgis, MD, Department of Psychiatry, Columbia University, College of Physicians \& Surgeons and New York State Psychiatric Institute, New York, USA; Michael R. Phillips, MD, Department of Psychiatry, Columbia University, College of Physicians \& Surgeons, New York, USA and Beijing Suicide Research and Prevention Center, Beijing Hui Long Guan Hospital, Beijing, China; Xiaodong Li, MS, Department Center, Beijing Hui Long Guan Hospital, Beijing, China; Xiaodong Li, MS, Department
of Psychiatry, Columbia University, College of Physicians \& Surgeons and New York State Psychiatric Institute, New York, USA; Kejin Li, Bachelor of Medicine, Beijing Suicide Research and Prevention Center, Beijing Hui Long Guan Hospital, Beijing, China; Huiping Jiang, PhD, Department of Psychiatry, Columbia University, College of Physicians \& Surgeons and New York State Psychiatric Institute, New York, USA; chengjing Wu, Bachelor of Medicine, Beijing Suicide Research and Prevention Center, Beiijing Hui Long Guan Hospital, Beiijing, China; Naihua Duan, PhD, Center, Beijing Hui Long Guan Hospital, Beijing, China; Naihua Duan, PhD,
Department of Psychiatry, Columbia University, College of Physicians \& Surgeons and New York State Psychiatric Institute, New York, USA; Yajuan Niu, Bachelor of Medicine, Beijing Suicide Research and Prevention Center, Beijing Hui Long Guan Hospital, Beijing, China; Jeffrey A. Lieberman, MD, Department of Psychiatry, Columbia University, College of Physicians \& Surgeons and New York State Psychiatric Institute, New York, USA

Correspondence: Jeffrey A. Lieberman, MD, College of Physicians and Surgeons, Columbia University, New York State Psychiatric Institute, 1051 Riverside Dr., Unit 4, New York, NY 10032, USA. Email: jlieberman@columbia.edu

First received 15 Apr 2010, final revision 24 Aug 2010, accepted 29 Sep 2010
}

\section{Funding}

This work was supported by funds from the Novartis Pharmaceutical Company, which also supplied medications.

\section{Acknowledgements}

We would like to acknowledge the support of the staff at the Beijing Suicide Research and Prevention Center and the Beijing Hui Long Guan Hospital and the participation of the patients who enrolled in this study.

\section{References}

1 McGlashan TH, Johannessen JO. Early detection and intervention with schizophrenia: rationale. Schizophr Bull 1996; 22: 201-22.

2 Wyatt RJ. Neuroleptics and the natural course of schizophrenia. Schizophr Bull 1991; 17: 325-51.

3 Emsley R, Oosthuizen PP, Kidd M, Koen L, Niehaus DJ, Turner HJ. Remission in first-episode psychosis: predictor variables and symptom improvement patterns. J Clin Psychiatry 2006; 67: 1707-12.

4 Marshall M, Lewis S, Lockwood A, Drake R, Jones P, Croudace T. Association between duration of untreated psychosis and outcome in cohorts of first-episode patients: a systematic review. Arch Gen Psychiatry 2005; 62: 975-83.

5 Perkins D, Lieberman J, Gu H, Tohen M, McEvoy J, Green A, et al. Predictors of antipsychotic treatment response in patients with first-episode schizophrenia, schizoaffective and schizophreniform disorders. $\mathrm{Br} \mathrm{J}$ Psychiatry 2004; 185: 18-24.

6 Perkins DO, Gu H, Boteva K, Lieberman JA. Relationship between duration of untreated psychosis and outcome in first-episode schizophrenia: a critical review and meta-analysis. Am J Psychiatry 2005; 162: 1785-804.
7 Lieberman JA, Tollefson GD, Charles C, Zipursky R, Sharma T, Kahn RS, et al. Antipsychotic drug effects on brain morphology in first-episode psychosis. Arch Gen Psychiatry 2005; 62: 361-70.

8 Lieberman JA, Bymaster FP, Meltzer HY, Deutch AY, Duncan GE, Marx CE, et al. Antipsychotic drugs: comparison in animal models of efficacy, neurotransmitter regulation, and neuroprotection. Pharmacol Rev 2008; 60 358-403.

9 Crespo-Facorro B, Perez-Iglesias R, Ramirez-Bonilla M, Martinez-Garcia O, Llorca J, Vazquez-Barquero JL. A practical clinical trial comparing haloperidol, risperidone, and olanzapine for the acute treatment of first-episode nonaffective psychosis. J Clin Psychiatry 2006; 67: 1511-21.

10 Emsley RA. Risperidone in the treatment of first-episode psychotic patients: a double-blind multicenter study. Risperidone Working Group. Schizophr Bull 1999; 25: 721-9.

11 Moller HJ, Riedel M, Jager M, Wickelmaier F, Maier W, Kuhn KU, et al. Shortterm treatment with risperidone or haloperidol in first-episode schizophrenia: 8 -week results of a randomized controlled trial within the German Research Network on Schizophrenia. Int J Neuropsychopharmacol 2008; 11: 985-97.

12 Lieberman JA, Tollefson G, Tohen M, Green Al, Gur RE, Kahn R, et al. Comparative efficacy and safety of atypical and conventional antipsychotic drugs in first-episode psychosis: a randomized, double-blind trial of olanzapine versus haloperidol. Am J Psychiatry 2003; 160: 1396-404.

13 Sanger TM, Lieberman JA, Tohen M, Grundy S, Beasley C Jr, Tollefson GD. Olanzapine versus haloperidol treatment in first-episode psychosis. Am J Psychiatry 1999; 156: 79-87.

14 Gaebel W, Riesbeck M, Wolwer W, Klimke A, Eickhoff M, von Wilmsdorff M, et al. Maintenance treatment with risperidone or low-dose haloperidol in first-episode schizophrenia: 1-year results of a randomized controlled trial within the German Research Network on Schizophrenia. J Clin Psychiatry 2007; 68: 1763-74.

15 Schooler N, Rabinowitz J, Davidson M, Emsley R, Harvey PD, Kopala L, et al. Risperidone and haloperidol in first-episode psychosis: a long-term randomized trial. Am J Psychiatry 2005; 162: 947-53.

16 Green Al, Lieberman JA, Hamer RM, Glick ID, Gur RE, Kahn RS, et al. Olanzapine and haloperidol in first episode psychosis: two-year data. Schizophr Res 2006; 86: 234-43.

17 Kahn RS, Fleischhacker WW, Boter H, Davidson M, Vergouwe Y, Keet IP, et al. Effectiveness of antipsychotic drugs in first-episode schizophrenia and schizophreniform disorder: an open randomised clinical trial. Lancet 2008; 371: 1085-97.

18 Lieberman JA, Stroup TS, McEvoy JP, Swartz MS, Rosenheck RA, Perkins DO, et al. Effectiveness of antipsychotic drugs in patients with chronic schizophrenia. N Engl J Med 2005; 353: 1209-23.

19 Jones PB, Barnes TR, Davies L, Dunn G, Lloyd H, Hayhurst KP, et al. Randomized controlled trial of the effect on Quality of Life of second- vs firstgeneration antipsychotic drugs in schizophrenia: Cost Utility of the Latest Antipsychotic Drugs in Schizophrenia Study (CUtLASS 1). Arch Gen Psychiatry 2006; 63: 1079-87.

20 Kane J, Honigfeld G, Singer J, Meltzer H. Clozapine for the treatment-resistant schizophrenic. A double-blind comparison with chlorpromazine. Arch Gen Psychiatry 1988; 45: 789-96.

21 Sikich L, Frazier JA, Mcclellan J, Findling RL, Vitiello B, Ritz L, et al. Doubleblind comparison of first- and second-generation antipsychotics in earlyonset schizophrenia and schizo-affective disorder: findings from the treatment of early-onset schizophrenia spectrum disorders (TEOSS) study. Am J Psychiatry 2008; 165: 1420-31.

22 Leucht S, Corves C, Arbter D, Engel RR, Li C, Davis JM. Second-generation versus first-generation antipsychotic drugs for schizophrenia: a metaanalysis. Lancet 2009; 373: 31-41.

23 Breier A, Hamilton SH. Comparative efficacy of olanzapine and haloperidol for patients with treatment-resistant schizophrenia. Biol Psychiatry 1999; 45: 403-11.

24 Tollefson GD, Birkett MA, Kiesler GM, Wood AJ. Double-blind comparison of olanzapine versus clozapine in schizophrenic patients clinically eligible for treatment with clozapine. Biol Psychiatry 2001; 49: 52-63.

25 Lieberman JA, Phillips M, Gu H, Stroup S, Zhang P, Kong L, et al. Atypical and conventional antipsychotic drugs in treatment-naive first-episode schizophrenia: a 52-week randomized trial of clozapine vs chlorpromazine. Neuropsychopharmacology 2003; 28: 995-1003.

26 Phillips MR, Xiong W, Zhao ZA. Issues Involved in the Use of Scales for the Assessment of Negative and Positive Symptoms in Psychotic Patients [in Chinese]. Hubei Science and Technology Publishing House, 1990.

27 First MB, Spitzer RL, Gibbon M, Williams JBW. Structured Clinical Interview for DSM-IV Axis I Disorders, Clinician Version (SCID-CV). American Psychiatric Press, 1996 
28 Phillips MR, Xiong W, Wang RW, Gao YH, Wang XQ, Zhang NP. Reliability and validity of the Chinese versions of the Scales for Assessment of Positive and Negative Symptoms. Acta Psychiatr Scand 1991; 84: 364-70.

29 Guy W. ECDEU Assessment Manual for Psychopharmacology. Revised DHEW Pub. (ADM). National Institute for Mental Health, 1976.

30 Jones SH, Thornicroft G, Coffey M, Dunn G. A brief mental health outcome scale - reliability and validity of the Global Assessment of Functioning (GAF). Br J Psychiatry 1995; 166: 654-9.

31 Simpson GM, Angus JW. A rating scale for extrapyramidal side effects. Acta Psychiatr Scand Suppl 1970; 212: 11-9.

32 Simpson GM, Lee JH, Zoubok B, Gardos G. A rating scale for tardive dyskinesia. Psychopharmacology (Berl) 1979; 64: 171-9.

33 Little RJA, Rubin DB. Statistical Analysis with Missing Data. John Wiley \& Sons, 2002.

34 Agid O, Remington G, Kapur S, Arenovich T, Zipursky RB. Early use of clozapine for poorly responding first-episode psychosis. J Clin Psychopharmacol 2007; 27: 369-73.

35 Gitlin M, Nuechterlein K, Subotnik KL, Ventura J, Mintz J, Fogelson DL, et al. Clinical outcome following neuroleptic discontinuation in patients with remitted recent-onset schizophrenia. Am J Psychiatry 2001; 158: 1835-42.
36 Robinson D, Woerner MG, Alvir JM, Bilder R, Goldman R, Geisler S, et al. Predictors of relapse following response from a first episode of schizophrenia or schizoaffective disorder. Arch Gen Psychiatry 1999; 56: 241-7.

37 Szymanski SR, Cannon TD, Gallacher F, Erwin RJ, Gur RE. Course of treatment response in first-episode and chronic schizophrenia. Am J Psychiatry 1996; 153: $519-25$.

38 Lehman AF, Lieberman JA, Dixon LB, McGlashan TH, Miller AL, Perkins DO, et al. Practice guideline for the treatment of patients with schizophrenia, second edition. Am J Psychiatry 2004; 161: 1-56.

39 Penn DL, Waldheter EJ, Perkins DO, Mueser KT, Lieberman JA. Psychosocial treatment for first-episode psychosis: a research update. Am J Psychiatry 2005; 162: 2220-32.

40 Kane JM. Tardive dyskinesia rates with atypical antipsychotics in adults: prevalence and incidence. J Clin Psychiatry 2004; 65 (suppl 9): 16-20.

41 Alvir JM, Lieberman JA, Safferman AZ, Schwimmer JL, Schaaf JA. Clozapine-induced agranulocytosis. Incidence and risk factors in the United States. N Engl J Med 1993; 329: 162-7.

42 Allison DB, Mentore JL, Heo M, Chandler LP, Cappelleri JC, Infante MC, et al. Antipsychotic-induced weight gain: a comprehensive research synthesis. Am J Psychiatry 1999; 156: 1686-96.

43 Newcomer JW. Antipsychotic medications: metabolic and cardiovascular risk Clin Psychiatry. 2007; 68 (suppl 4): 8-13.

\section{0 \\ Reading for Well-Being Project}

\section{David Fearnley}

Shared reading, aloud, is about getting more out of great writing. This innovative approach, developed by the Reader Organisation in Merseyside, has been very successful when tried in mental health settings. A group of patients and a trained facilitator will read afresh poems, short stories or novels (often a 'classic'). Expertise is sought from the literature, not the therapist. Increased confidence, concentration and self-development are commonly reported outcomes. Sharing the reading experience can empower many patients who may not have achieved their educational potential. Engagement can also be developed by promoting an interest in reading from within the healthcare team.

Some of the books we have read are: Emily Brontë's Wuthering Heights, Alan Bennett's The Uncommon Reader, John Steinbeck's Of Mice and Men, George Orwell's Animal Farm - all went down well! 